\title{
DEVELOPMENT OF THE PAPILLARY MUSCLES OF THE MITRAL VALVE: MORPHOGENETIC BACKGROUND OF PARACHUTE-LIKE ASYMMETRIC MITRAL VALVES AND OTHER MITRAL VALVE ANOMALIES
}

Petra W. Oosthoek, PhD

Arnold C. G. Wenink, MD, PhD

Lambertus J. Wisse

Adriana C. Gittenberger-de Groot, $\mathrm{PhD}$
Objectives: To understand papillary muscle malformations, such as in parachute mitral valves or parachute-like asymmetric mitral valves, we studied the development of papillary muscles. Methods: Normal human hearts at between 5 and 19 weeks of development were studied with immunohistochemistry, three-dimensional reconstructions, and gross inspection. Scanning electron microscopy was used to study human and rat hearts. Results: In embryonic hearts a prominent horseshoe-shaped myocardial ridge runs from the anterior wall through the apex to the posterior wall of the left ventricle. In the atrioventricular region this ridge is continuous with atrial myocardium and covered with cushion tissue. The anterior and posterior parts of the trabecular ridge enlarge and loosen their connections with the atrial myocardium. Their lateral sides gradually delaminate from the left ventricular wall, and the continuity between the two parts is incorporated in the apical trabecular network. In this way the anterior and posterior parts of the ridge transform into the anterolateral and the posteromedial papillary muscles, respectively. Simultaneously, the cushions remodel into valve leaflets and chordae. Only the chordal part of the cushions remains attached to the developing papillary muscles. Conclusions: Disturbed delamination of the anterior or posterior part of the trabecular ridge from the ventricular wall, combined with underdevelopment of chordae, seems to be the cause of asymmetric mitral valves. Parachute valves, however, develop when the connection between the posterior and anterior part of the ridge condenses to form one single papillary muscle. Thus parachute valves and parachute-like asymmetric mitral valves originate in different ways. (J Thorac Cardiovasc Surg 1998; 116:36-46)
A recent investigation of the anatomy of parachute valves and parachute-like asymmetric mitral valves $^{1}$ motivated the study of mitral valve papillary muscle development. Both these mitral valve anom-

From the Department of Anatomy, Leiden University Medical Center, Leiden, The Netherlands.

Financial support of The Netherlands Heart Foundation, grant 93.057, is acknowledged.

This work was performed at the Department of Anatomy, Leiden University Medical Center, The Netherlands.

Received for publication Nov. 17, 1997; revisions requested Feb. 4, 1998; revisions received March 2, 1998; accepted for publication March 2, 1998.

Address for reprints: P. W. Oosthoek, PhD, Department of Anatomy, Leiden University, P.O. Box 9602, 2300 RC Leiden, The Netherlands.

Copyright (C) 1998 by Mosby, Inc.

0022-5223/98 \$5.00 + $0 \quad \mathbf{1 2 / 1 / 9 0 0 1 2}$ alies have unifocalization of chordae, but there are substantial differences in abnormal anatomy of the papillary muscles.

In parachute mitral valves, there is one single papillary muscle to which all chordae are attached. ${ }^{2}$ In parachute-like asymmetric mitral valves, however, two papillary muscles can be recognized, one of which has abnormal morphologic characteristics. ${ }^{1}$

We have found a morphologic spectrum of the abnormal papillary muscle in asymmetric mitral valves. In the most severe cases, (grade III), the abnormally long papillary muscle is attached over its entire length to the ventricular wall, with its tip located close to the atrioventricular anulus. The valve leaflets are directly attached to this papillary muscle because chordae are not present. In grade II asymmetry, a few short chordae are attached to an abnormal long papillary muscle. In grade I asymme- 
try, the abnormally long papillary muscle is also characteristic but chordal morphologic characteristics are close to normal.

Reports on papillary muscle formation ${ }^{3,4}$ are scarce and not detailed enough to explain the development of these two different papillary muscle malformations. Nor do they explain such other deviations as malattachment of papillary muscles or papillary muscles reaching to the atrioventricular anulus. To get more insight into the normal papillary muscle development and related congenital malformations of the mitral valve, we studied papillary muscle formation in the left ventricles of human hearts in embryonic and fetal stages, and papillary muscle morphology in neonatal and adult hearts. Scanning electron microscopic investigations of embryonic rat hearts were included in this study.

\section{Material and methods}

Tissue preparation. Normal human hearts were obtained from 28 embryos and fetuses at between 5 and 19 weeks of development. The embryos and fetuses were obtained after termination of pregnancies at the Center for Human Reproduction in Leiden, The Netherlands, and the Stimezo Clinic in The Hague, The Netherlands. Written informed consent was obtained for each subject, and the studies of human material were approved by the medical ethical committee of the Leiden University. According to the regulations given by the Leiden University Committee on Experimental Animals, eight pregnant Wistar rats underwent hysterectomy with ether anaesthesia and were killed by cervical dislocation. The rat embryos, ranging from 12 to 18 embryonic days, were used for scanning electron microscopy. These rat hearts were also used in a previous study of tricuspid valve development. ${ }^{5}$

Immunohistochemistry and gross inspection. Twentyfive human hearts were fixed overnight in a mixture of methanol, acetone, acetic acid, and water (7:7:7:5 volume ratios) at room temperature and dehydrated in a graded series of ethanol. Ten of these hearts were studied under the dissection microscope. The other 15 hearts were embedded in paraffin (Merck, Darmstadt, Germany) and prepared for immunohistochemical examination. These hearts were cut in sections of $5 \mu \mathrm{m}$ and mounted on glycerine-albumin-coated glass slides. After removal of the paraffin and rehydration of the sections, endogenous peroxidase was reduced by hydrogen peroxide $(0.3 \%$ volume/volume) in phosphate-buffered saline solution ( $\mathrm{pH} 7.3$ ) for 15 minutes. Mouse monoclonal antibodies against $\alpha$ - or $\beta$-myosin heavy chain ${ }^{6}$ kindly supplied by Prof. Dr. A. F. M. Moorman (University of Amsterdam, The Netherlands) were used to distinguish myocardial myocytes. Incubations were followed by a peroxidaselabeled rabbit antibody against mouse immunoglobulin (DAKO, Glostrup, Denmark), goat antirabbit immunoglobulin (Nordic, Tilburg, The Netherlands), and a rabbit peroxidase-antiperoxidase complex (Nordic). ${ }^{7}$ All antibodies were diluted in phosphate-buffered saline solution supplemented with ovalbumin ( $1 \%$ weight/volume) and polysorbate 20 (Tween-20, $0.05 \%$, volume/volume). Between all incubation steps, the sections were rinsed in phospate-buffered saline solution. Binding of the antibodies was made visible by 3,3'-diaminobenzidine (D8001; Sigma, Belgium) in $0.05 \mathrm{~mol} / \mathrm{L}$ Tris maleic acid (pH 7.6).

Three-dimensional reconstruction. By use of a projection microscope, the contours of the myocardium and cushion tissue in tissue sections of human hearts between 5 and 16 weeks of development were magnified and drawn on acetate sheets. ${ }^{8}$ The sheets were stacked with appropriate spacing so that a first three-dimensional impression of the left atrioventricular junction could be obtained. Graphic reconstructions ${ }^{9,10}$ were made of the human hearts, and these were compared with the scanning electron micrographs of the rat and human hearts.

Scanning electron microscopy. Three human hearts at 12,13 , and 14 weeks of development and 25 rat hearts at between 12 and 17 embryonic days were perfused with 500 IU thromboliquine (Organon Teknika, B.V., Boxtel, The Netherlands) in $100 \mathrm{ml}$ phosphate-buffered saline solution ( $\mathrm{pH}$ 7.3), followed by half-strength Karnovsky's fixative, ${ }^{11}$ into the right atrium. The procedure was continued by immersion fixation in half-strength Karnovsky's fixative and gross dissection of the heart under the microscope. The specimens were then rinsed in $0.1 \mathrm{~mol} / \mathrm{L}$ sodium cacodylate buffer ( $\mathrm{pH} 7.2$ ) and postfixed for 1 hour in $1 \%$ osmium tetroxide in the same buffer at room temperature, followed by dehydration in a graded ethanol series. The specimens were critical-point dried over carbon dioxide by conventional methods, sputter-coated with gold for 3 minutes (MED 010; Balzers, Balzers, Liechtenstein), and studied in the scanning electron microscope (SEM 525; Philips, Eindhoven, The Netherlands).

Adult human heart. In addition to the embryonic stages, we studied more than 150 human hearts, neonatal to adult, in search of variation of normal and abnormal morphologic characteristics of the mitral valve. These hearts were used in a previous study of parachute-like asymmetric mitral valves. ${ }^{1}$

\section{Results}

Horseshoe-shaped muscular ridge in left ventricle: 5 weeks. In the human heart at 5 weeks of development and in rat hearts at 13 embryonic days, the left ventricular wall was composed of two layers. The outer layer of the wall consisted of compact myocardium, whereas the inner layer contained many trabeculae (Fig. 1). Scanning electron microscopy of rat hearts at 13 embryonic days demonstrated a prominent muscular ridge that was composed of thick trabeculae, present within the inner layer (Fig. 1,b). This ridge was connected to the ventricular wall and ran from posterior in the atrioventricular junction through the apex to the anterior part of the atrioventricular junction. The ridge itself contained intertrabecular spaces and thus was not a compact structure. Of interest, more prominent 

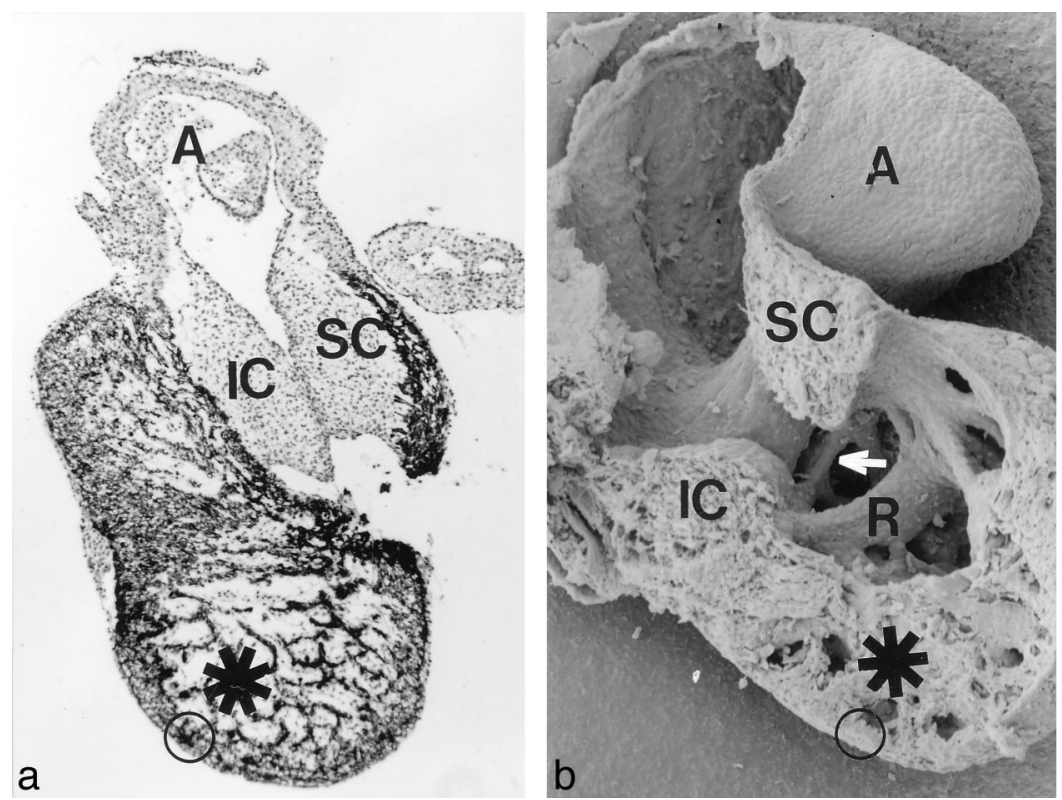

Fig. 1. a, Tissue section of human heart at 5 weeks of development, stained for $\beta$-myosin heavy chain. b, Scanning electron micrograph of rat heart at 13 embryonic days, demonstrating trabecular ridge $(R)$ in the left ventricle and some minor ridges at the parietal wall (arrow). In both panels, the compact outer layer (open circle) and the trabecular inner layer (asterisk) of the ventricular wall are visible. A, Atrium; SC, superior atrioventricular cushion; $I C$, inferior atrioventricular cushion.

trabeculae that ran from posterior to anterior were present lateral from and parallel to the main myocardial ridge (Fig. 1,b).

In the atrioventricular region, the posterior part of the main horseshoe-shaped ridge was covered by the inferior atrioventricular cushion and the anterior part was covered with the superior cushion. Also lateral from the main trabecular ridge, the myocardium of the atrioventricular junction was covered by cushion tissue.

Formation of aortic leaflet of mitral valve, connecting posterior and anterior parts of ridge: 7 to 71/2 weeks. A similar horseshoe-shaped trabecular ridge was observed in reconstructions of a human heart at 7 weeks of development (Fig. 2,a). In tissue sections, it was demonstrated that the myocardium of the posterior and anterior parts of the ridge was continuous with atrial myocardium in the atrioventricular junction (Fig. 2,b). Fusion of the inferior and superior atrioventricular cushions was almost complete, but a fusion line was still visible in the middle of the cushion tissue between the anterior and posterior parts of the trabecular ridge (Fig. 2, a). This atrioventricular cushion tissue, connecting the anterior and posterior parts of the trabecular ridge, formed the aortic leaflet of the mitral valve (the first leaflet of the mitral valve that could be recognized). Underneath the cushion tissue, which was present lateral to the main trabecular ridge, gaps became visible in the myocardium of the parietal ventricular wall (Fig. 2, b). This process is called delamination of ventricular myocardium in the literature.

A few days later in the human heart, at $71 / 2$ weeks of development, the trabecular ridge was still prominent; however, the anterior and posterior parts of the ridge were relatively long compared with the distance between these two parts of the ridge (not shown). The intertrabecular spaces within the ridge were less obvious at this stage, giving it a more compact appearance. The aortic leaflet of the mitral valve, between the anterior and superior parts of the ridge, was relatively small, but the fusion line between the former inferior and superior cushions could no longer be discriminated.

Loosening of ridge from left ventricular wall to become freely movable papillary muscles: 8 to 10 weeks. In the human heart between 8 and 10 weeks of development and in the rat heart between 15 and 17 embryonic days, the trabecular ridge remained recognizable in the left ventricle. However, the anterior and posterior parts were rather long com- 

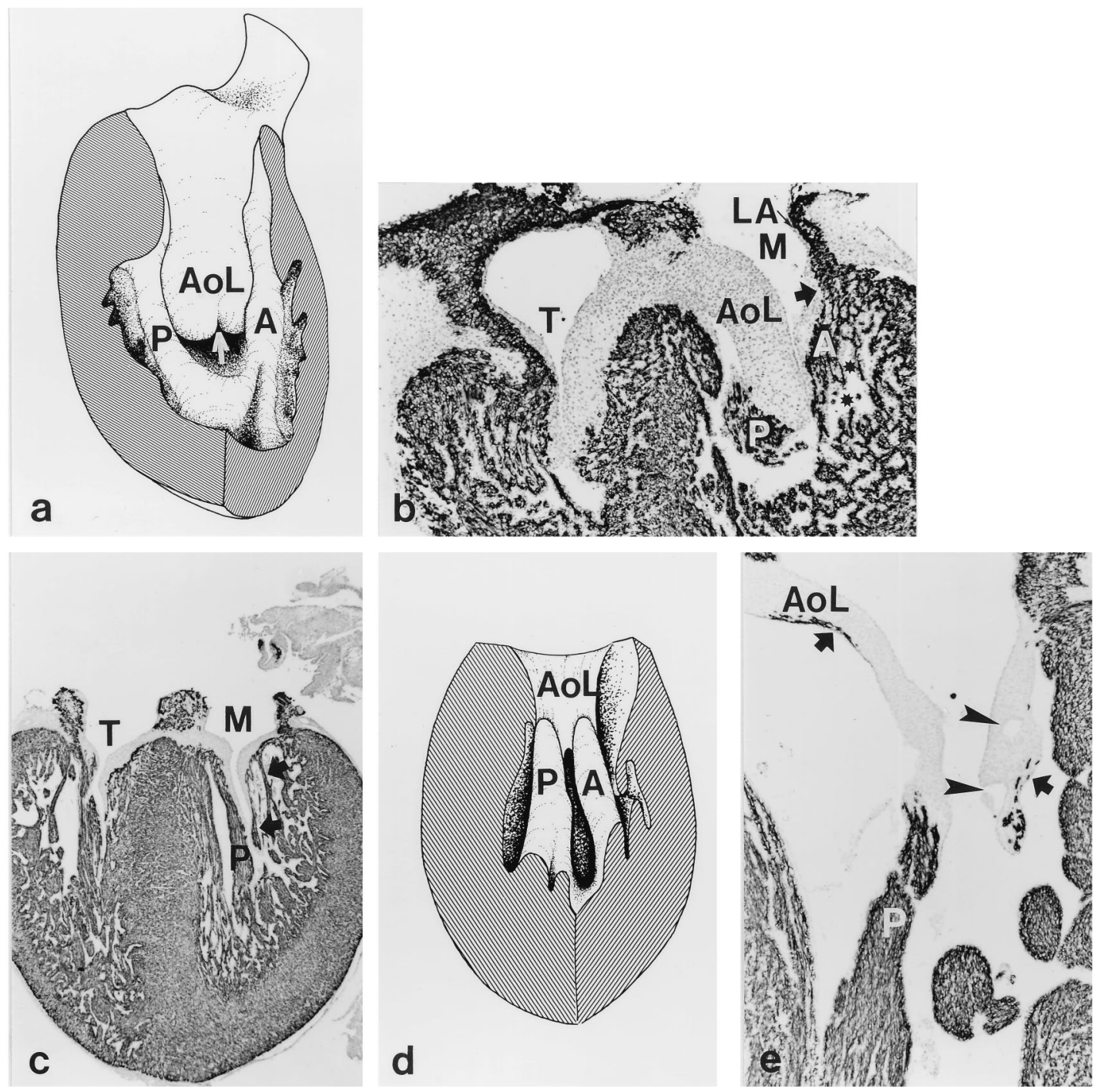

Fig. 2. a, Three-dimensional reconstruction of human heart at 7 weeks of development, showing horseshoe-shaped muscular ridge. Developing aortic leaflet of mitral valve $(A o L)$ is present between posterior $(P)$ and anterior $(A)$ parts of the ridge. The fusion line between the two cushions (arrow) is still visible at this stage. $\mathbf{b}$, Tissue section of same heart as in $\mathbf{a}$, stained for $\alpha$ - and $\beta$-myosin heavy chain, in which continuity of the anterior part of the ridge $(A)$ and the atrial myocardium $(L A)$ is indicated by a thick arrow. At this stage the first signs of delamination of myocardium are visible (asterisks). c, Tissue section of human heart at 8 weeks of development. Continuity between myocardium of the atrium, the ventricle, and the developing papillary muscles $(P)$ is still obvious. Trabeculae (arrows) are present between delaminated myocardium and left ventricular wall. d, Three-dimensional reconstruction of human heart at 10 weeks of development. Trabecular ridge is now quite recognizable as developing posteromedial $(P)$ and anterolateral $(A)$ papillary muscles. e, Tissue section of same heart as in $\mathbf{d}$, stained for $\alpha$ - and $\beta$-myosin heavy chain. Developing posteromedial papillary muscle $(P)$ is attached to left ventricular wall only at its base and to cushion tissue at its tip. The delaminated myocardial layer is disrupted (arrows). Arrowheads point to small gaps within cushion tissue. $M$, Mitral valve; $T$, tricuspid valve.

pared with the distance between them. In the apex, the myocardial continuity between the anterior and superior parts of the ridge was still present, but this continuity was incorporated more within the trabecular network and was therefore less pronounced. 

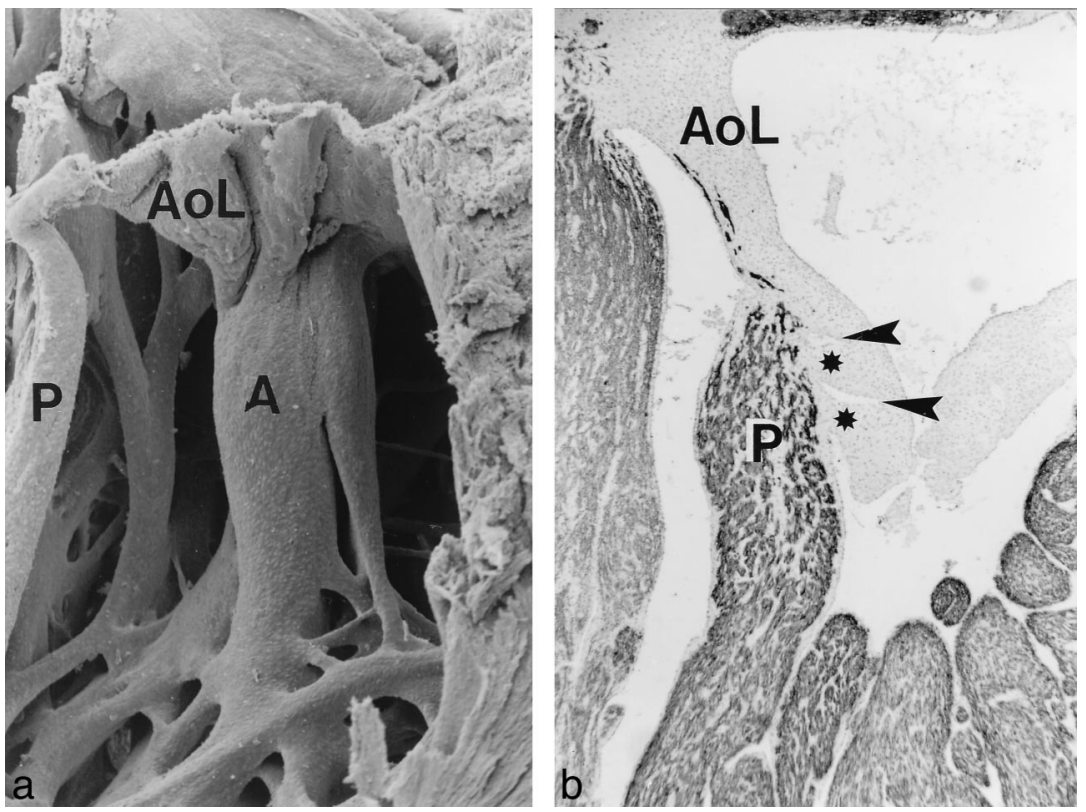

Fig. 3. a, Scanning electron micrograph of human heart at 12 weeks of development, in which continuity of developing papillary muscles and trabeculae in the ventricle is visible. Cushion tissue of developing aortic leaflet of mitral valve $(A o L)$ is directly attached to anterolateral $(A)$ and posteromedial $(P)$ papillary muscles. b, Tissue section of human heart at $12 \frac{1}{2} 2$ weeks of development, stained for actin. The aortic leaflet of mitral valve $(A o L)$ is directly attached to posteromedial papillary muscle $(P)$; however, developing interchordal spaces (arrowheads) and chordae (asterisks) are already present.

At 8 weeks the myocardial ridge was still connected through some trabeculae over its entire length to the ventricular wall. The connecting trabeculae were less obvious in a heart at 9 weeks of development, and at 10 weeks the trabecular connections between the anterior and posterior parts of the ridge and the ventricular wall were no longer visible. The posterior and anterior parts of the ridge now had the morphologic characteristics of small posteromedial and anterolateral papillary muscle respectively; they were freely movable because they were attached to the ventricular wall only at their bases (Fig. 2, $d$ and $e$ ).

In human hearts at 8 weeks of development, many muscular connections were visible not only between the developing papillary muscles but also between the delaminated ventricular myocardium underneath the cushion tissue lateral from the main myocardial ridge and the parietal part of the ventricular wall (Fig. 2,c). Whether these trabeculae were also horseshoe-shaped bundles, as in the rat heart at 13 embryonic days, could not be observed in the reconstructions. At 9 weeks of development, the myocardial connections between the delaminated myocardium and the parietal part of the left ventricular free wall could still be observed, but myocardial bundles to the apex were no longer present. At 10 weeks of development, these connections were observed only in the atrioventricular region, and they disappeared after that age. As a result, in the atrioventricular junction the cushion tissue of the developing mural leaflet was connected only to the atrial and ventricular myocardium, and more apically to the tips of the two papillary muscles (Fig. 2,e).

The continuity between the myocardium of the ridge and the atrial myocardium, which was still present at 8 weeks of development, was disrupted at 10 weeks of development (Fig. 2,e). Only remnants of the layer of delaminated myocardium underneath the cushion tissue were visible. From 10 weeks of development onward, the myocardium of the trabecular ridges was thus connected to the myocardium only in the atrioventricular region by the cushion tissue of the developing aortic and mural leaflets of the mitral valve.

At 10 weeks of development, within the cushion tissue of both developing valve leaflets (the aortic 

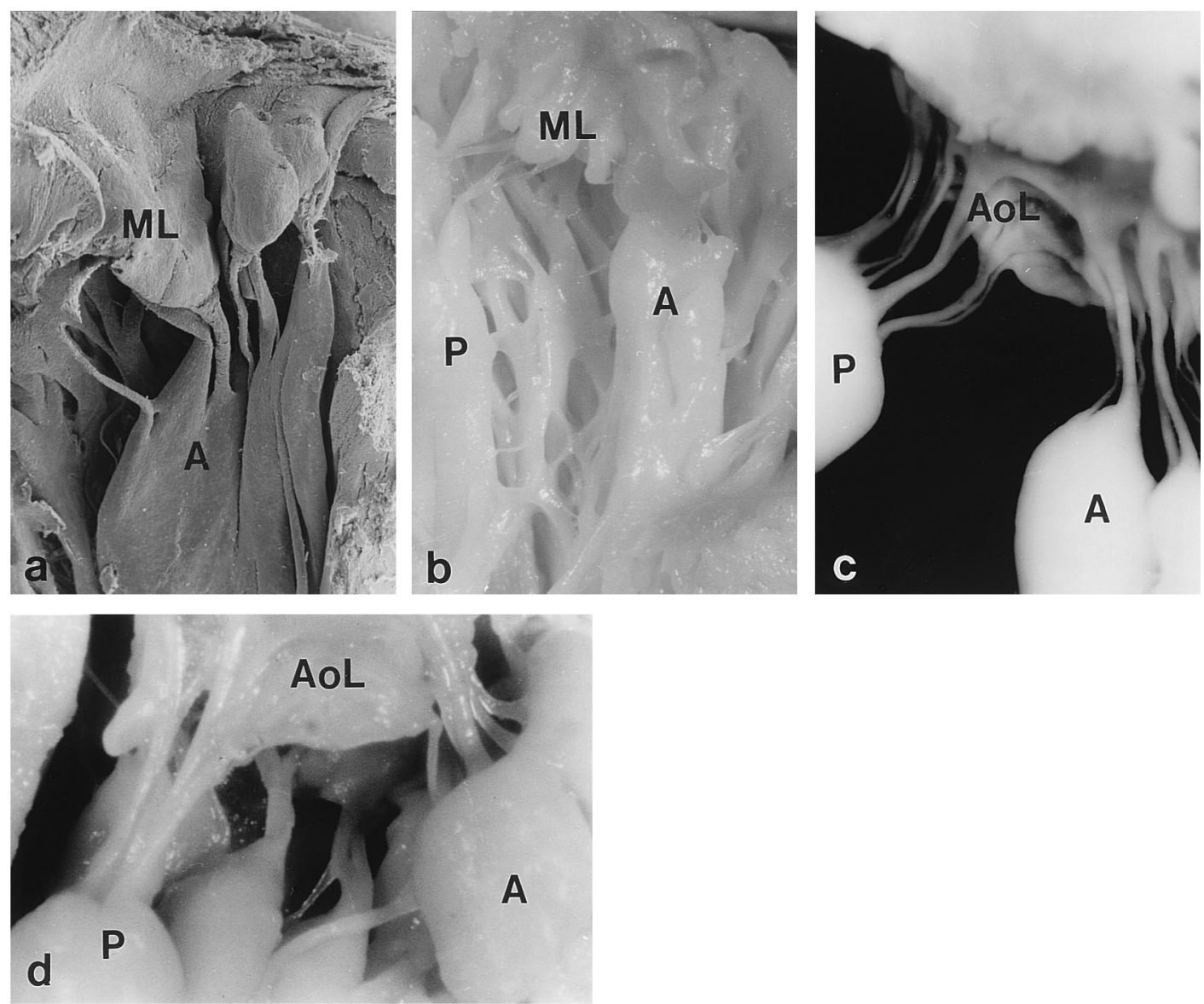

Fig. 4. a, Scanning electron micrograph of human heart at 14 weeks of development. b, Human heart at 15 weeks of development. c, Human heart at 17 weeks of development. d, Human heart at 18 weeks of development. Panels demonstrate interindividual differences in papillary muscle morphologic characteristics, such as long papillary muscles (a and b) or muscles with two (c) or more (d) bellies. A, Anterolateral papillary muscle; $A o L$, aortic leaflet of mitral valve; $M L$, mural leaflet; $P$, posteromedial papillary muscle.

and mural leaflets of the mitral valve), small gaps were visible in the area where the cushion tissue was connected to the tips of the developing papillary muscles (Fig. 2,e).

Freely movable papillary muscles, formation of chordae: 11 to 13 weeks. In scanning electron micrographs of a heart at 12 weeks of development, the two papillary muscles were quite recognizable in the left ventricular lumen (Fig. 3, a). Some intertrabecular spaces remained within the papillary muscles, especially at their bases, where the continuity with the ventricular trabecular network was obvious. Two valve leaflets could be discriminated at this stage that were directly attached to the papillary muscles. Separate chordae were not yet visible, but the gaps in the cushion tissue at the tips of the papillary muscles were more obvious in tissue sections of hearts of compara- ble age than in the heart at 10 weeks of development. The cushion tissue between the gaps was still connected to the papillary muscles and resembled extremely short chordae (Fig. 3, b).

Complete valves with two papillary muscles, two leaflets, and chordae: 14 to 19 weeks. At 14 weeks of development and later, the two papillary muscles were clearly present. In tissue sections it was obvious that the chordae were composed of cushion tissue, and the chordae could be discriminated with both the scanning electron microscope and the dissection microscope. Although the valves were only a few millimeters in size from about 15 weeks of development onward, the morphologic characteristics of the entire mitral valves (fibrous leaflets and chordae and two papillary muscles), were comparable to those in the adult heart. 


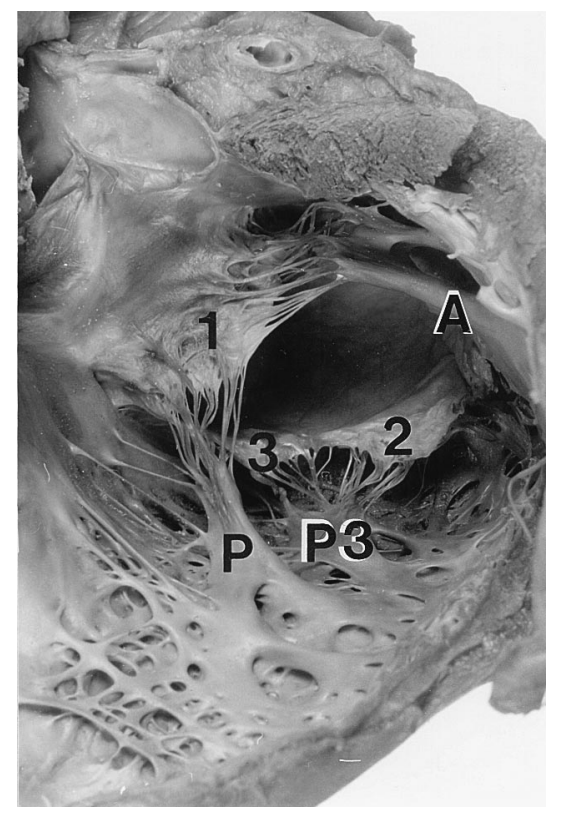

Fig. 5. Abnormal adult mitral valve with three papillary muscles. $A$, Anterolateral papillary muscle; $P$, posteromedial papillary muscle; $P 3$, third papillary muscle at the parietal wall; 1 , aortic leaflet of mitral valve; 2 and 3 , two leaflets at the parietal wall.

Interestingly, from 14 weeks of development onward, we observed interindividual differences in papillary muscle morphologic characteristics. Variants included relatively long papillary muscles and papillary muscles with two or more bellies (Fig. 4).

Abnormal variation: Adult mitral valve with three papillary muscles. We observed one case of special interest among the newborn and adult heart specimens in which, apart from the anterolateral and posteromedial papillary muscle, a third papillary muscle was present (Fig. 5). This additional papillary muscle was attached to the parietal wall in the left ventricle, at the location where the myocardial connections between cushion tissue and ventricular wall normally disappear.

\section{Discussion}

In embryonic human and rat hearts, we observed a myocardial horseshoe-shaped ridge in the left ventricle. This ridge could be traced in consecutive stages of development and appeared to transform into the papillary muscles of the mitral valve (Fig. 6 ). The transformation implied gradual loosening of the muscular ridge from the left ventricular wall, starting in the atrioventricular region. Furthermore, during growth of the left ventricle, the distance between the anterior and posterior parts of the ridge enlarged and the continuity in the apex was incorporated in the apical trabecular network, so that two separate papillary muscles could be discriminated that were attached only at their bases to the ventricular wall. Simultaneously, the cushion tissue gradually loosed contact with the myocardium of the ridge, with the exception of those parts of the cushion tissue that transformed into the chordae. Logically, these developing chordae remained attached to the papillary muscles. The process of papillary muscle development is comparable between rats and human beings but takes about 17 weeks in the human heart and only about 17 days in the rat heart. Another difference is that the papillary muscles of adult rodent hearts are relatively long and attached at their base and lateral side to the left ventricular wall. ${ }^{12}$

Loosening from the left ventricular wall, as we found for the development of papillary muscles, is a similar process to that described in the literature for the valve leaflets and can therefore also be referred to as delamination. ${ }^{13-15}$ Our observations confirm the suggestions that fusion of trabeculae ${ }^{13}$ plays a role in the formation of solid papillary muscles. Condensation of trabeculae ${ }^{16}$ and loosening of ventricular myocardium ${ }^{17}$ have also been observed in chicken hearts. Unfortunately, the cellular mechanisms behind these phenomena are still not understood.

The continuity of the components that contribute to the mitral valve-cushion tissue, trabeculae, and ventricular wall-was prominent in the scanning electron micrographs. This continuity has been described previously and was nicely illustrated by Morse $^{18}$ in the chick heart. The borderline between cushion tissue and myocardium, however, can only be observed in tissue sections. In contradiction to reports in the literature, in which it has been assumed that the chordae develop from myocardium, ${ }^{13-15,18-20}$ we concluded that both the leaflets and the chordae originate from cushion tissue. Our conclusion is supported by a study of extracellular matrix components, in which we found that the immunohistochemical characteristics of cushions and chordae are the same. ${ }^{21}$

In fetal hearts at between 14 and 19 weeks of development, we observed interindividual differences in papillary muscle morphologic characteristics, as have been described in normal adult human hearts by Ranganathan and Silver. ${ }^{22}$ The origin of tethered papillary muscles and distinct "bellies" can 


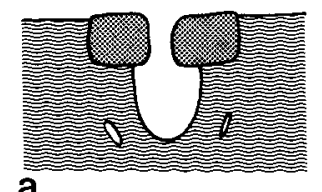

a

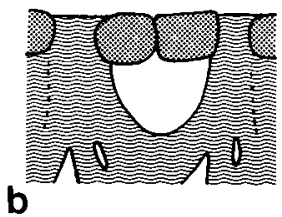

C
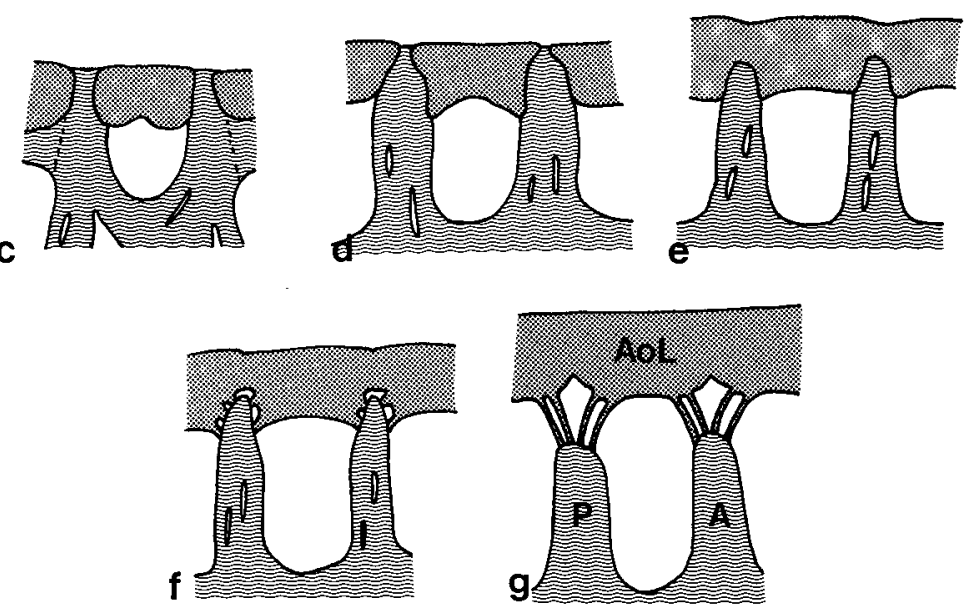

Fig. 6. Schematic representation of developing papillary muscles of the mitral valve, with its aortic leaflet between them. Horseshoe-shaped trabecular ridge (stripes) is present; it gradually loosens from the left ventricular wall. Meanwhile, cushion tissue (dots) transforms into leaflets and chordae. a, 5 weeks of development; b, 7 weeks; c, 71/2 weeks; d, 8 weeks; e, 10 weeks; f, 12 weeks; g, 14 weeks. AoL, Aortic leaflet of mitral valve; $P$, posteromedial papillary muscle; $A$, anterolateral papillary muscle.

be understood by realizing that the papillary muscles are initially attached over their entire length to the ventricular wall and that they are composed of a group of condensed trabeculae.

Not only variations in normal development but also abnormal papillary muscles can be explained by the process of development. A third papillary muscle at the lateral wall, as we found in an adult heart, seems to originate during the embryonic period when the trabeculae at the lateral wall also condense into a papillary muscle. This situation is normal for the chicken heart. ${ }^{23}$

In view of a previous study, ${ }^{1}$ we were especially interested in the developmental background of parachute mitral valves and parachute-like asymmetric mitral valves. In a series of asymmetric mitral valves, a spectrum of abnormal morphologic characteristics of one papillary muscle was found. ${ }^{1}$ The abnormal papillary muscle was elongated, located higher in the left ventricle, with its tip reaching to the anulus, and attached at both its base and lateral side to the left ventricular wall. Interestingly, the spectrum of abnormal morphologic characteristics is also observed in successive stages during normal development of the papillary muscles. It is therefore plausible that asymmetry is a persistent embryonic situation that is caused by a disturbed delamination of either the anterior or the posterior part of the trabecular ridge from the ventricular wall (Fig. 7, $a$ through $g$ ).

A true parachute mitral valve, one the other hand, has only one papillary muscle, which is centrally located in the left ventricle. This papillary muscle is not attached at its lateral side to the ventricular wall. $^{2}$ In other words, the true parachute valve seems to have a different origin than that of the parachute-like asymmetric mitral valve. The term fused papillary muscles has been used for parachute mitral valves, ${ }^{24}$ but we think that this term is confusing in view of the developmental background. Realizing that the papillary muscles develop from one continuous trabecular ridge, we assume that parachute mitral valves are the result of persistence of this connection during delamination of the trabecular ridge from the ventricular wall (Fig. 7, $a$ and $h$ through $k$ ). The description underdeveloped 


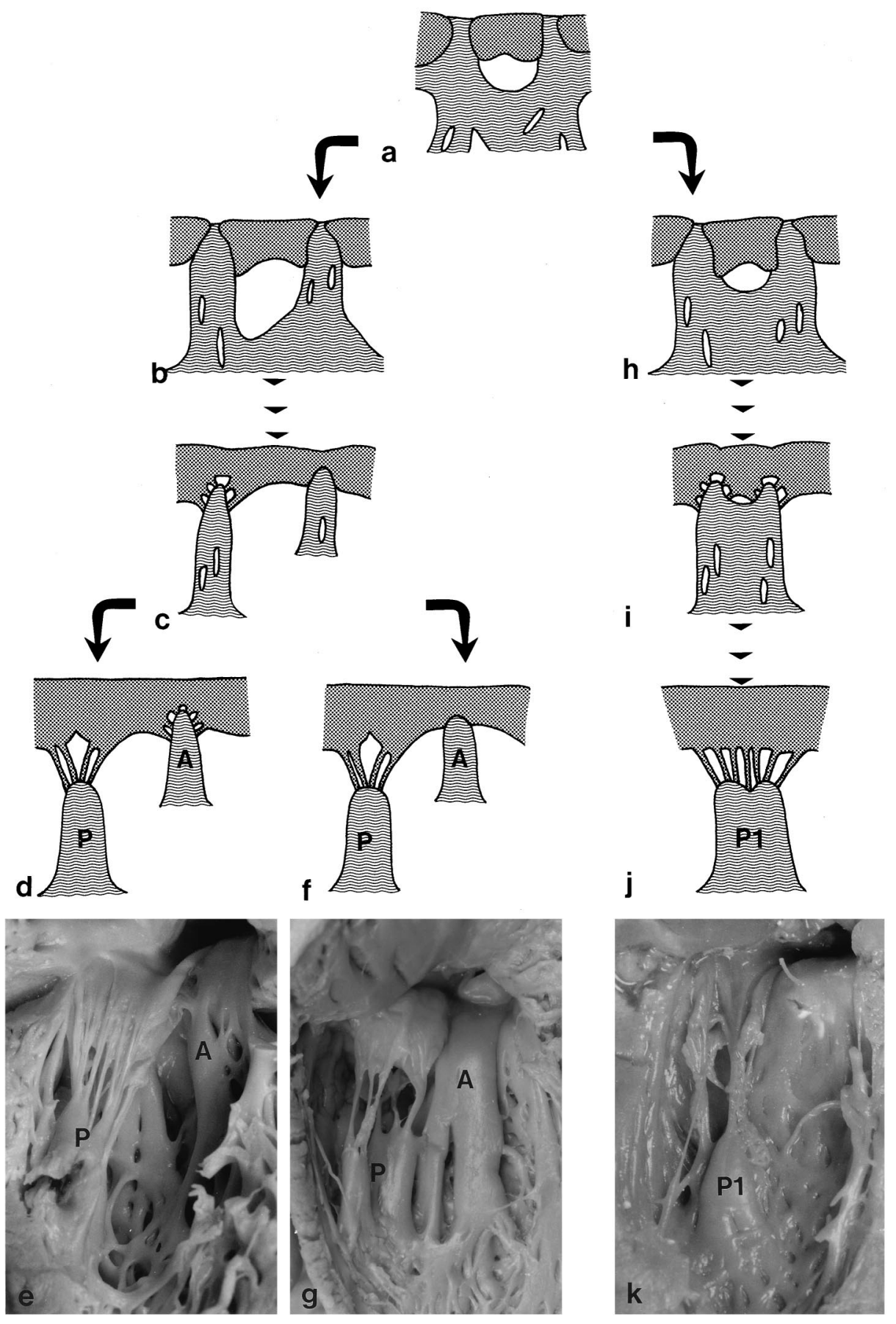

Fig. 7. Hypothetic development of parachute-like asymmetric mitral valves (a through $\mathbf{g}$ ) and parachute mitral valves ( $\mathbf{a}$ and $\mathbf{h}$ through $\mathbf{k}$ ). Asymmetry develops if one of two papillary muscles does not delaminate from left ventricular wall and its tip remains in the atrioventricular region. When chordae develop only partially, grade II asymmetry results (d and $\mathbf{e}$ ). If chordae do not develop at all, valve leaflets remain directly attached to the papillary muscle, resulting in grade III asymmetry (f and $\mathbf{g}$ ). In parachute mitral valve, anterior and posterior parts of horseshoe-shaped muscular ridge do not incorporate on the apical trabecular network but remain connected to each other and grow out into one single papillary muscle (h through k). $P$, Posteromedial papillary muscle; $A$, anterolateral papillary muscle; $P 1$, single papillary muscle. 
space $^{25}$ between the two papillary muscles thus seems to be in better agreement with the developmental background of parachute mitral valves. Unfortunately, parachute mitral valves are rare, and we have only one specimen in our collection. We were unable to verify the developmental background by a spectrum of abnormal papillary muscles, as we were able to do for the parachute-like asymmetric mitral valves. ${ }^{1}$

Not only in asymmetric mitral valves but also in other malformations, one or two of the papillary muscles have remained abnormally long and connected to the ventricular wall and valve leaflets. Examples include outflow tract obstructions caused by one ${ }^{26}$ or two ${ }^{27}$ papillary muscles and a papillary muscle that reaches to the atrioventricular anulus. ${ }^{15}$ In all these cases, the papillary muscle morphologic character can be explained by an incomplete delamination of the trabecular ridge from the left ventricular wall, a disturbed loosening of the cushion tissue, and underdevelopment of chordae.

Disturbances in papillary muscle formation may also cause abnormal accessory connections of atrioventricular conduction. ${ }^{28}$ These connections can be explained by persistence of the continuity of atrioventricular myocardium with the myocardium of the papillary muscles. This is a normal situation until 8 weeks of development, but the myocardial connection normally disappears then, so that only the cushion tissue of the developing leaflets and chordae forms a bridge between atrioventricular myocardium and papillary muscles. In fact, the presence of accessory pathways between atrial and ventricular myocardium in the atrioventricular junction is a comparable malformation. These connections persist if the atrial and ventricular myocardium is not separated by cushion tissue of the atrioventricular valve anulus. $^{29}$

Not only is detailed knowledge of normal papillary muscle formation important to understand when and how various papillary muscle malformations develop, it is also of relevance in the early detection of these anomalies. At present such examination can only be performed in postmortem material. In view of improvements being made in the current technique of fetal echocardiography, ${ }^{30}$ however, prenatal evaluation of the papillary muscles should become possible in the near future.

We thank Monica Mentink for technical assistance and Jan Lens for the photographic work.

\section{REFERENCES}

1. Oosthoek PW, Wenink ACG, Macedo AJ, Gittenberger-de Groot AC. The parachute-like asymmetric mitral valve and its two papillary muscles. J Thorac Cardiovasc Surg 1997;114: 9-15.

2. Shone JD, Sellers RD, Anderson RC, Adams P, Lillehei CW, Edwards JE. The developmental complex of "parachute mitral valve," supravalvular ring of left atrium, subaortic stenosis, and coarctation of aorta. Am J Cardiol 1963;714-25.

3. Ho SY, Angelini A, Moscoso G. Developmental cardiac anatomy. In: Lamsback WJ, editor. Fetal and neonatal cardiology. Philadelphia: Saunders; 1990. p. 3-16.

4. Wenink ACG, Gittenberger-de Groot AC. Embryology of the mitral valve. Int J Cardiol 1986;11:75-84.

5. Wenink ACG, Wisse BJ, Groenendijk PM. Development of the inlet portion of the right ventricle in the embryonic rat heart: the basis for tricuspid valve development. Anat Rec 1994;239:216-23.

6. Wessels A, Vermeulen JL, Virágh S, Kálmán F, Lamers WH, Moorman AF. Spatial distribution of "tissue-specific" antigens in the developing human heart and skeletal muscle. III. An immunohistochemical analysis of the distribution of the neural tissue antigen GIN2 in the embryonic heart; implications for the development of the atrioventricular conduction system. Anat Rec 1991;229:355-68.

7. Sternberger LA. Immunohistochemistry. New York: Wiley; 1986. p. 90-209.

8. de Jong F, Geerts WJC, Lamers WH, Los JA, Moorman AFM. Isomyosin expression patterns in tubular stages of chicken heart development: a 3-D immunohistochemical analysis. Anat Embryol 1987;177:81-90.

9. Tinkelenberg J. Graphic reconstruction, microanatomy with a pencil. J Audiov Media Med 1979;2:102-6.

10. Wenink ACG, Chon Y. The value of graphic reconstructions: comparison with scanning electron microscopy. Anat Rec 1984;210:537-40.

11. Karnovsky MJ. A formaldehyde-glutaraldehyde fixative of high osmolarity for use in electron microscopy. J Cell Biol 1965;27:137A-8A.

12. Icardo JM, Arrechedera H, Colvee E. The atrioventricular valves of the mouse. I. A scanning electron microscope study. J Anat 1993;182:87-94.

13. van Mierop LHS, Alley RD, Kausel HW, Stranahan A. The anatomy and embryology of endocardial cushion defects. J Thorac Cardiovasc Surg 1962;43:71-83.

14. Lamers WH, Virágh S, Wessels A, Moorman AF, Anderson RH. Formation of the tricuspid valve in the human heart. Circulation 1995;91:111-21.

15. Wenink ACG, Gittenberger-de Groot AC, Brom AG. Developmental considerations of mitral valve anomalies. Int J Cardiol 1986;11:85-98.

16. Ben-Shachar G, Arcilla RA, Lucas RV, Manasek FJ. Ventricular trabeculations in the chick embryo heart and their contribution to ventricular and muscular septal development. Circ Res 1985;57:759-66.

17. Icardo JM, Fernandez-Terán A. Morphologic study of ventricular trabeculation in the embryonic chick heart. Acta Anat 1987;130:264-74.

18. Morse DE, Hamlett WC, Noble CW. Morphogenesis of chordae tendineae. I: Scanning electron microscopy. Anat Rec 1984;210:629-38. 
19. Odgers PNB. The development of the atrio-ventricular valves in man. J Anat 1939;73:643-57.

20. Bouchey D, Argraves WS, Little CD. Fibulin-1, vitronectin, and fibronectin expression during avian cardiac valve and septa development. Anat Rec 1996;244:540-51.

21. Oosthoek PW, Wenink ACG, Vrolijk BC, Wisse LJ, DeRuiter MC, Poelmann RE, et al. Development of the atrioventricular valve tension apparatus in the human heart. Anat Embryol. In press 1998.

22. Ranganathan N, Silver MD. The mitral valve in man: a review of anatomy and its clinical significance. Anat Clin 1981;2:361-7.

23. Lu Y, James TN, Bootsma M, Terasaki F. Histological organization of the right and left atrioventricular valves of the chicken heart and their relationship to the atrioventricular Purkinje ring and the middle bundle branch. Anat Rec 1993;235:74-86

24. Anderson RH, Macartney FJ, Shinebourne EA, Tynan M. Mitral valve anomalies and supravalvar mitral ring. In: Anderson RH, Macartney FJ, Shinebourne EA, Tynan M, editors. Pediatric cardiology. New York: Churchill Livingstone; 1987. p. 1023-56.

25. Rosenquist GC. Congenital mitral valve disease associated with coarctation of the aorta: a spectrum that includes parachute deformity of the mitral valve. Circulation 1974;49: 985-93.

26. Wada Y, Kawai T, Oga K, Oka T. Left ventricular outflow tract obstruction and mitral regurgitation caused by papillary muscle abnormalities: a case report. J Thorac Cardiovasc Surg 1993;106:1223-5.

27. Klues HG, Roberts WC, Maron BJ. Anomalous insertion of papillary muscle directly into anterior mitral leaflet in hypertrophic cardiomyopathy: significance in producing left ventricular outflow obstruction. Circulation 1991;84:1188-97.

28. Sealy WC. Surgical anatomy of accessory connections of atrioventricular conduction. Ann Thorac Surg 1994;57:167583.

29. Wessels A, Markman MW, Vermeulen JL, Anderson RH, Moorman AFM, Lamers WH. The development of the atrioventricular junction in the human heart. Circ Res 1996; 78:110-7.

30. Allan LD. Echocardiographic detection of congenital heart disease in the fetus: present and future. Br Heart J 1995;74: 103-6.

Disclaimer. The above study used aborted human fetuses. The Journal does not wish to be involved in the ethics of abortion because this is clearly outside its field of competence. To that end, the Editor ascertained from the author the fact that the decision to have an abortion by the mother was made without her knowledge that the fetus might be used for research. Only after consent for abortion was obtained was consent for the proposed research on the fetus requested.

Editor

\section{Bound volumes available to subscribers}

Bound volumes of The Journal of Thoracic and Cardiovascular Surgery are available to subscribers (only) for the 1998 issues from the Publisher, at a cost of $\$ 122.00$ for domestic, $\$ 151.94$ for Canadian, and $\$ 142.00$ for international subscribers for Vol. 115 (January-June) and Vol. 116 (July-December). Shipping charges are included. Each bound volume contains a subject and author index and all advertising is removed. Copies are shipped within 60 days after publication of the last issue of the volume. The binding is durable buckram with the Journal name, volume number, and year stamped in gold on the spine. Payment must accompany all orders. Contact Mosby, Inc., Subscription Services, 11830 Westline Industrial Drive, St. Louis, Missouri 63146-3318, USA; phone 800-453-4351 or 314-453-4351.

Subscriptions must be in force to qualify. Bound volumes are not available in place of a regular Journal subscription. 